\title{
Integrability analysis of the partial differential equation describing the classical bond-pricing model of mathematical finance
}

https://doi.org/10.1515/phys-2018-0096

Received May 17, 2018; accepted June 19, 2018

\begin{abstract}
The invariant approach is employed to solve the Cauchy problem for the bond-pricing partial differential equation (PDE) of mathematical finance. We first briefly review the invariant criteria for a scalar secondorder parabolic PDE in two independent variables and then utilize it to reduce the bond-pricing equation to different Lie canonical forms. We show that the invariant approach aids in transforming the bond-pricing equation to the second Lie canonical form and that with a proper parametric selection, the bond-pricing PDE can be converted to the first Lie canonical form which is the classical heat equation. Different cases are deduced for which the original equation reduces to the first and second Lie canonical forms. For each of the cases, we work out the transformations which map the bond-pricing equation into the heat equation and also to the second Lie canonical form. We construct the fundamental solutions for the bond-pricing model via these transformations by utilizing the fundamental solutions of the classical heat equation as well as solution to the second Lie canonical form. Finally, the closed-form analytical solutions of the Cauchy initial value problems for the bond-pricing model with proper choice of terminal conditions are obtained.
\end{abstract}

Keywords: Bond-pricing equation; Invariant method; Fundamental solutions; Cauchy problem for the bond-pricing model

PACS: 02.20.Sv; 02.30.Jr; 04.20.Jb

\footnotetext{
*Corresponding Author: Taha Aziz: School of Computer, Statistical and Mathematical Sciences, North-West University, Potchefstroom Campus, Private Bag X2520, Potchefstroom, South Africa, E-mail: tahaaziz77@yahoo.com, Tel.: +27-18-2852494

Aeeman Fatima, Chaudry Masood Khalique: International Institute for Symmetry Analysis and Mathematical Modeling, Department of Mathematical Sciences, North-West University, Mafikeng Campus, Private Bag X2046, Mmabatho 2735, South Africa
}

\section{Introduction}

Lie symmetry analysis has been widely applied in the study of differential equations since the 60 s of the last century although it was inaugurated by the Norwegian mathematician Sophus Lie (1842-1899) in the later part of the nineteenth century. Symmetry methods are particularly employed to obtain group-invariant solutions and reduction of differential equations. In recent years symmetry methods have been applied to a number of problems of science and engineering. The main advantage of these methods is that they can be successfully applied to nonlinear differential equations.

The applications of Lie theory to financial mathematics is relatively new. Generally, the differential equations that arise in mathematical finance are usually solved by adhoc methods or by using numerical computation. On the other hand, the Lie group theory allows us to find complete symmetry groups that may be exploited to find closed form solutions or reductions of the differential equations. Regarding the application of Lie group theory to some financial mathematics and economics problems, we mention here the existence of several recent works. One of the first examples of Lie theory used in finance was due to Gazizov and Ibragimov [1], who studied the famous BlackScholes equation. The analysis of various forms of the Black-Scholes equation using symmetry analysis is performed by Tamizhmani et al. [2]. The algebraic structure of the classical optimal investment-consumption problem is investigated from the perspective of Lie group theory by Motsepa et al. [3]. The group classification of the general bond-option pricing equation is carried out by Motsepa et al. [4]. Lo and Hui [5] used different techniques based on Lie algebras to deal with the valuation of financial derivatives. Sinkala [6] recently studied the arbitrage-free stock price models using the symmetry based approach. The fundamental solutions to the Cauchy problem for zerocoupon bond-pricing equations have been investigated by Pooe et al. [7]. Application of Lie theory to economics is due to Basov [8], who described some methods based on Lie 
groups in order to solve the multidimensional screening problem. The option pricing models with dividend yields was investigated via Lie theory by Liu and Wang [9]. Wang et al. [10] utilized the Lie group analysis method to the geometric average Asian option pricing equation. The group invariant solution of solution of the Black-Scholes equation for the price of a (European) call option using symmetry method is obtained by Davison and Mamba [11]. Dimas et al. [12] investigated some of the well-known equations that arise in mathematics of finance, such as BlackScholes, Longtsaff, Vasicek and CIR equations. Lie point symmetries of these equations were found and their exact solutions were deduced in [12]. In recent times, the group approach has been widely applied to some other problems of mathematical finance. See, for example, Naicker et al. [13], Polidoro [14], Sinkala et al. [15], Zhou and Xiao [16] and Bordag and Yamshchikov [17].

In this paper, we study the general bond-pricing equation

$$
\frac{\partial u}{\partial t}+\frac{1}{2} \rho^{2} y^{2 \gamma} \frac{\partial^{2} u}{\partial y^{2}}+\left(\alpha+\beta y-\lambda \rho y^{\gamma}\right) \frac{\partial u}{\partial y}-y u=0,
$$

where $\alpha, \beta, \rho, \lambda$ and $\gamma$ are constants, $t$ is time, $y$ is the stock price or instantaneous short-term interest rate at current time $t$ and $u(y, t)$ is the current value of the bond. The short-term interest $y$ follows a stochastic process of the form

$$
d y=(\alpha+\beta y) d t+\beta y^{\gamma} d W(t),
$$

where $W(t)$ is the Wiener process. The parameter $\lambda$ is called the market price of risk.

The theory of bond-pricing began in 1900 when the French mathematician Bachelier [17] deduced the bondpricing on the assumption that stock prices follow a Brownian motion. Later the works of Merton [18] and Black and Scholes [19] also opened a new era in mathematical modelling of problems in finance. These models have been formulated in terms of stochastic differential equations and after certain assumptions are written in the form of linear parabolic PDEs with constant or variable coefficients. The first bond-pricing equation was introduced by Vasicek [20] and thereafter many researchers, for example, $[21,22]$ came up with various modifications.

The study of bond-pricing PDE (1) is very important as many one-factor interest rate models that have been proposed in the literature can be nested in (2) with the precise forms of the arbitrary elements depending upon the particular model under consideration. For example, Vasicek [20] $(\gamma=0)$, Cox-Ingersoll and Ross [23] $(\gamma=1 / 2)$, Brennan and Schwartz [24] $(\gamma=1)$ and Dothan [25] $(\alpha=\beta=$ $0, \gamma=1$ ) are special cases of the PDE (1).
A Lie symmetry group is used to construct symmetries of the underlying differential equations. Lie was the first who initiated the group classification of differential equations. In fact, Lie classified scalar linear secondorder PDEs in two independent variables and developed a method for their integration. One of the earlier studies that proposed the invariant approach to Fokker-Planck equations was made by Johnpillai and Mahomed [26]. Semiinvariants for the $(1+1)$ linear parabolic equations with two independent variables and one dependent variable were derived in [26]. In addition, a joint invariant equation was obtained for the linear parabolic equation and the $(1+1)$ linear parabolic equation was reducible via a local equivalence transformation to the one-dimensional heat equation. They also proved the necessary and sufficient conditions for a scalar linear $(1+1)$ parabolic equation, in terms of the coefficient of the equation, to be reducible via equivalence transformations to the classical heat equation. Later, Mahomed [27] provided the complete invariant characterization of a scalar linear $(1+1)$ parabolic PDE of one space variable and one time variable into four canonical forms. The Lie group approach is therefore one of the powerful tools to find the fundamental solutions of PDEs. The fundamental solution of a PDE can be used to find more solutions of the PDE by convolution. However, the symmetry methods provide a systematic approach to the problem of finding fundamental solutions of PDEs. Countless difficult problems for PDEs are found in the literature for which the fundamental solutions are found. Such solutions play an important role in providing the benchmark for the numerical solutions of the PDEs.

We know that the bond-pricing PDE (1) is a linear parabolic PDE, therefore we employ the invariant approach to elegantly classify it. The symmetry group classification of PDE (1) was performed in Sinkala et al. [28]. In our study, we demonstrate that this can also be performed in a simpler way using the invariant approach. We find some new cases for which the bond-pricing equation is transformed into the classical heat equation. We also deduce that the bond-pricing equation admits four nontrivial symmetries and hence can be reduced to the second Lie canonical form. Moreover, we determine the transformations which reduce the bond-pricing equation into different Lie canonical forms. These transformations are further utilized to obtain various new exact solutions of the bond-pricing equation. The closed-form solution of the Cauchy initial value problem of the bond-pricing equation is also obtained.

The layout of the paper is as follows. In the next section, we briefly recall the salient features of the invariant criteria for scalar (1+1) parabolic PDEs as provided in [27]. 
In Section 3 we apply the invariant approach to the bondpricing PDE (1) and compute some exact fundamental solutions of the problem. Section 4 present solutions to the Cauchy value problems. Some concluding remarks are presented in Section 5.

\section{Invariant approach for scalar linear (1+1) parabolic equations}

In this section we briefly recall main results of [27] on the invariant characterization of the scalar linear $(1+1)$ parabolic PDE

$$
u_{t}=a(t, x) u_{x x}+b(t, x) u_{x}+c(t, x) u,
$$

where $a, b$ and $c$ are continuous functions of $t$ and $x$. The equivalence transformations [29] of the parabolic PDE (3) are an infinite group which comprises linear transformations of the dependent variable given by

$$
\bar{u}=\sigma(t, x) u, \quad \sigma \neq 0
$$

and invertible transformations of independent variables

$$
\bar{t}=\phi(t), \quad \bar{x}=\psi(t, x), \quad \dot{\phi} \neq 0, \psi_{x} \neq 0,
$$

where $\phi, \psi$ and $\sigma$ are arbitrary functions with restrictions for invertibility of the transformations and $\bar{u}$ is the new dependent variable. Two linear parabolic PDEs of the form (3) are equivalent to each other if one can be mapped to the other by appropriate combinations of the equivalence transformations (4) and (5). Lie [21] proved that the scalar linear parabolic PDE (3) has the following four canonical forms:

$$
\begin{aligned}
& u_{t}=u_{x x}, \\
& u_{t}=u_{x x}+\frac{A}{x^{2}} u, \quad A \neq 0, \\
& u_{t}=u_{x x}+c(x) u, \quad c \neq 0, A / x^{2}, \\
& u_{t}=u_{x x}+c(t, x) u, c \neq 0, A / x^{2} .
\end{aligned}
$$

The heat equation, which is the first Lie canonical form, has six nontrivial symmetries as well as the infinite number of trivial superposition symmetries. The second Lie canonical form has four nontrivial symmetries. The third in general has two symmetries and the fourth has one nontrivial symmetry in general.

We now state the following theorems $[26,27]$ which provide an invariant criteria for reduction of scalar linear (1+1) parabolic PDE (3) into the Lie canonical forms (6).
Theorem 1. The scalar linear (1+1) parabolic PDE (3) is reducible via equivalence transformations (4) and (5) to the heat equation

$$
\bar{u}_{\bar{t}}=\bar{u}_{\bar{x} \bar{x}}
$$

if and only if the coefficients of the parabolic PDE (3) satisfies the invariant condition

$$
2 L_{X}+2 M_{X}-N_{X}=0
$$

where

$$
\begin{aligned}
& L=|a|^{\frac{1}{2}}\left[|a|^{\frac{1}{2}} J_{x}\right]_{x}, \quad M=|a|^{\frac{1}{2}}\left[|a|^{\frac{1}{2}} \partial_{t}(b / 2 a)\right]_{x} . \\
& N=|a|^{\frac{1}{2}} \partial_{t}^{2}\left(1 /|a|^{\frac{1}{2}}\right)
\end{aligned}
$$

with J given by

$$
J=c-\frac{b_{x}}{2}+\frac{b a_{x}}{2 a}+\frac{a_{x x}}{4}-\frac{3}{16} \frac{a_{x}^{2}}{a}-\frac{a_{t}}{2 a}-\frac{b^{2}}{4 a} .
$$

Theorem 2. The scalar linear (1+1) parabolic PDE (3) is reducible to the second Lie canonical form

$$
\bar{u}_{\bar{t}}=\bar{u}_{\bar{x} \bar{x}}+\frac{A}{\bar{x}^{2}} \bar{u},
$$

where $A$ is a nonzero constant, if and only if the coefficients of the parabolic PDE (3) satisfy the invariant equation, provided that condition (8) does not hold,

$$
\begin{aligned}
& 20 L_{X}+20 M_{X}-10 N_{X}+10\left[|a|^{\frac{1}{2}} M_{X}\right]_{X} \int \frac{d x}{|a|^{\frac{1}{2}}} \\
& -5\left[|a|^{\frac{1}{2}} N_{X}\right]_{X} \int \frac{d x}{|a|^{\frac{1}{2}}}+10\left[|a|^{\frac{1}{2}} L_{X}\right]_{X} \int \frac{d x}{|a|^{\frac{1}{2}}} \\
& +\left[|a|^{\frac{1}{2}}\left[|a|^{\frac{1}{2}} L_{X}\right]_{X}\right]_{X}\left(\int \frac{d x}{|a|^{\frac{1}{2}}}\right)^{2} \\
& +\left[|a|^{\frac{1}{2}}\left[|a|^{\frac{1}{2}} M_{X}\right]_{X}\right]_{X}\left(\int \frac{d x}{|a|^{\frac{1}{2}}}\right)^{2} \\
& -\frac{1}{2}\left[|a|^{\frac{1}{2}}\left[|a|^{\frac{1}{2}} N_{X}\right]_{X}\right]_{X}\left(\int \frac{d x}{|a|^{\frac{1}{2}}}\right)^{2}=0,
\end{aligned}
$$

where $L, M, N$ and J are as given in (9) and (10).

Theorem 3. The scalar linear (1+1) parabolic equation (3) which does not satisfy the conditions of Theorems 1 and 2 is equivalent to the third Lie canonical form

$$
\bar{u}_{\bar{t}}=\bar{u}_{\bar{x} \bar{x}}+\bar{c}(\bar{x}) \bar{u}
$$

if and only if the coefficients of parabolic equation (3) satisfy the invariant criterion, provided that the conditions (8) and (12) do not apply,

$$
\frac{\partial}{\partial t}\left[J+\partial_{t} \int \frac{b}{2 a} d x-\frac{1}{2} \int \frac{1}{|a|^{\frac{1}{2}}}\left(\partial_{t}^{2} \int \frac{d x}{|a|^{\frac{1}{2}}}\right) d x\right]=0 .
$$


Theorem 4. The scalar linear (1+1) parabolic equation (3) is equivalent to the fourth Lie canonical form

$$
\bar{u}_{\bar{t}}=\bar{u}_{\bar{x} \bar{x}}+\bar{c}(\bar{x}, \bar{t}) \bar{u}
$$

if and only if the coefficients of PDE (3) satisfy the invariant condition, provided that the conditions (8) and (12) do not hold,

$$
\frac{\partial}{\partial t}\left[J+\partial_{t} \int \frac{b}{2 a} d x-\frac{1}{2} \int \frac{1}{|a|^{\frac{1}{2}}}\left(\partial_{t}^{2} \int \frac{d x}{|a|^{\frac{1}{2}}}\right) d x\right] \neq 0 .
$$

Theorem 5. The linear parabolic equation (3) is reducible to the classical heat PDE $\bar{u}_{\bar{t}}=\bar{u}_{\bar{x} \bar{x}}$ via the transformations

$$
\begin{aligned}
\bar{t} & =\phi(t), \\
\bar{x} & = \pm \int\left[\dot{\phi} a(t, x)^{-1}\right]^{\frac{1}{2}} d x+\beta(t), \\
\bar{u} & =v(t)[a(t, x)]^{-\frac{1}{4}} u \exp \left[\int \frac{b(t, x)}{2 a(t, x)} d x\right. \\
& -\frac{1}{8} \frac{\ddot{\phi}}{\dot{\phi}}\left(\int \frac{d x}{a(t, x)^{\frac{1}{2}}}\right)^{2}-\frac{1}{2} \int \frac{1}{a(t, x)^{\frac{1}{2}}} \partial_{t} \\
& \left.\left(\int \frac{d x}{a(t, x)^{\frac{1}{2}}}\right) d x \pm \frac{1}{2} \frac{\dot{\beta}}{\dot{\phi}^{\frac{1}{2}}} \int \frac{d x}{a(t, x)^{\frac{1}{2}}}\right],
\end{aligned}
$$

where $\dot{\phi}$ and a have the same sign, and $\phi, \beta$, and $v$ satisfy

$$
\begin{aligned}
\dot{\phi} \bar{c} & =J+\partial_{t} \int \frac{b(t, x)}{2 a(t, x)} d x-\frac{1}{2} \int \frac{1}{a(t, x)^{\frac{1}{2}}} \partial_{t}^{2} \\
& \left(\int \frac{d x}{a(t, x)^{\frac{1}{2}}}\right) d x+f(t)\left(\int \frac{d x}{a(t, x)^{\frac{1}{2}}}\right)^{2} \\
& +g(t)\left(\int \frac{d x}{a(t, x)^{\frac{1}{2}}}\right)+h(t)
\end{aligned}
$$

in which $J$ is as in Eq. (10) and

$$
\begin{aligned}
& f(t)=\frac{1}{16} \frac{\ddot{\phi}^{2}}{\dot{\phi}^{2}}-\frac{1}{8}\left(\frac{\ddot{\phi}}{\dot{\phi}}\right)_{t}, \\
& g(t)= \pm \frac{1}{4} \frac{\ddot{\phi}}{\dot{\phi}} \frac{\dot{\beta}}{\dot{\phi}^{\frac{1}{2}}} \pm \frac{1}{2}\left(\frac{\dot{\beta}}{\dot{\phi}^{\frac{1}{2}}}\right)_{t}, \\
& h(t)=\frac{1}{4} \frac{\ddot{\phi}}{\dot{\phi}}+\frac{1}{4} \frac{\dot{\beta}^{2}}{\dot{\phi}}+\frac{\dot{v}}{v} .
\end{aligned}
$$

The functions $f, g$, and $h$ are constrained by the relation defined in Eq. (18). For reductions into the other Lie canonical forms, the transformation relations are derived in [27].

\section{Invariant solutions of bond-pricing equation}

In this section we apply the above theorems to the bondpricing PDE (1). We use the invariant approach encapsu- lated in the theorems above to simply and elegantly map the PDE (1) to the Lie canonical forms.

\subsection{Reduction to heat equation}

With the comparison of PDE (1) to the scalar linear $(1+1)$ parabolic PDE (12), the coefficients $a(t, y), b(t, y)$ and $c(t, y)$ are written as

$$
\begin{aligned}
& a(t, y)=-\frac{1}{2} \rho^{2} y^{2 \gamma}, \\
& b(t, y)=-\left(\alpha+\beta y-\lambda \rho y^{\gamma}\right), \\
& c(t, y)=y .
\end{aligned}
$$

By making use of Theorem 1, the values of the parameter $\lambda$ for which the PDE (1) can be mapped to the Lie canonical forms are obtained. First we evaluate $J$ as given in Eq. (10) for the bond-pricing PDE (1). Indeed it is

$$
\begin{aligned}
J= & y+\frac{1}{2} \beta-\beta \gamma+\frac{1}{2} \lambda^{2}+\frac{1}{2} \lambda \rho \gamma y^{\gamma-1}-\frac{1}{8} \rho^{2} \gamma^{2} y^{2 \gamma-2} \\
& +\frac{1}{4} \rho^{2} \gamma y^{2 \gamma-2}-\alpha \gamma y^{-1}+\frac{1}{2} \frac{\alpha^{2}}{\rho^{2}} y^{-2 \gamma}+\frac{\alpha \beta}{\rho^{2}} y^{1-2 \gamma} \\
& -\frac{\alpha \lambda}{\rho} y^{-\gamma}-\frac{\beta \lambda}{\rho} y^{1-\gamma}+\frac{\beta^{2}}{2 \rho^{2}} y^{2-2 \gamma} .
\end{aligned}
$$

The coefficients of the bond-pricing PDE (1) are independent of $t$, therefore we have

$$
M=N=0 .
$$

Thus the invariant condition (8) for reduction to the heat equation becomes

$$
L_{y}=0 .
$$

Making use of Eqs. (9), (20) and (21) into Eq. (23), we have

$$
\begin{aligned}
& \frac{1}{2} \rho^{2} \gamma(2 \gamma-1) y^{2 \gamma-2}+\frac{3}{2} \lambda \rho^{3} \gamma(\gamma-1)^{3} y^{3 \gamma-4} \\
& -\frac{3}{2} \rho^{4} \gamma^{2}(\gamma-1)^{3} y^{4 \gamma-5}+3 \rho^{4} \gamma(\gamma-1)^{3} y^{4 \gamma-5} \\
& +\frac{1}{2} \alpha \rho^{2} \gamma(\gamma-2)(2 \gamma-3) y^{2 \gamma-4}-\alpha^{2} \gamma(\gamma+1) y^{-3} \\
& +\frac{1}{2} \alpha \beta \gamma(1-2 \gamma) y^{-2}-\frac{1}{2} \alpha \lambda \rho \gamma(\gamma-2) y^{\gamma-3}=0 .
\end{aligned}
$$

We observe from condition (24) that all powers of $y$ cannot be distinct, otherwise we arrive at the condition $\rho=0$, which is inadmissible. Therefore, we only need to consider the values of the parameters for which the powers of $y$ are the same. As an example, if $2 \gamma-2=3 \gamma-4$, we immediately have $\gamma=2$ and the equating of separate powers of $y$ to zero in condition (24) yields $\alpha=0$ and $\lambda=-1 / \rho$. Similarly we determine all other non-trivial cases for which the invariant condition (24) holds. We arrive at the following cases 
for which the bond-pricing PDE (1) is reducible to classical heat equation.

Case I: $\gamma=2, \alpha=0, \lambda=-1 / \rho, \beta$ and $\rho$ are arbitrary

Case II: $\gamma=0, \alpha, \beta, \lambda$ and $\rho$ are arbitrary

Case III: $\gamma=1 / 2, \alpha=\rho^{2} / 4, \lambda, \beta$ and $\rho$ are arbitrary

Case IV: $\gamma=1 / 2, \alpha=3 \rho^{2} / 4, \lambda=0, \beta$ and $\rho$ are arbitrary

\subsection{Transformation formulae and fundamental solutions}

Here we find the transformations which reduce the bondpricing PDE (1) to the heat equation for each of the cases found in the previous subsection. Then these transformations are further utilized to obtain the fundamental solutions of bond-pricing equation.

\subsubsection{Case I: $\gamma=2, \alpha=0, \lambda=-1 / \rho, \beta$ and $\rho$ are arbitrary.}

Making use of the coefficients defined in Eq. (20) in Eqs. (18) and (19) in Theorem 5, we have

$$
\begin{aligned}
f(t) & =-\frac{\beta^{2}}{4}, \\
h(t) & =\frac{3 \beta}{2}-\frac{1}{2 \rho^{2}}, \\
\phi(t) & =\frac{C_{1}}{\beta} \tan (\beta t), \\
\beta(t) & =\frac{-\sqrt{2 C_{1}}}{\beta} \frac{1}{\cos (\beta t)}+\frac{\sqrt{C_{1}} C_{2}}{\beta} \tan (\beta t)+C_{3}, \\
g(t) & =\frac{\beta}{\sqrt{2}}, \\
v(t) & =\exp \left[-\frac{1}{2} \ln \cos (\beta t)-\frac{1}{2 \beta}\{(\tan (\beta t)-\beta t)\right. \\
& \left.\left.-\frac{2 \sqrt{2} C_{2}}{\beta} \frac{1}{\cos (\beta t)}+\frac{C_{2}^{2}}{\beta} \tan (\beta t)\right\}+h t\right],
\end{aligned}
$$

where $C_{1}, C_{2}$ and $C_{3}$ are constants of integration. Using the values of the functions from Eq. (25) into Eq. (17) and simplifying, we obtain the following transformations that reduce the parabolic equation (1) into the heat equation:

$$
\begin{aligned}
\bar{t}(y, t)= & \frac{C_{1} \tan (\beta t)}{\beta}, \\
\bar{y}(y, t)= & \pm \frac{\sqrt{2 C_{1}} y^{1-\gamma}}{\cos (\beta t) \rho(1-\gamma)}-\frac{\sqrt{2 C_{1}}}{\beta \cos (\beta t)} \\
& +\frac{\sqrt{C_{1}} C_{2} \tan (\beta t)}{\beta}+C_{3}, \\
\bar{u}(y, t)= & \left(\frac{1}{2} \rho^{2} y^{2 \gamma}\right)^{-\frac{1}{4}} \exp \left[-\frac{1}{2} \ln \cos (\beta t)\right.
\end{aligned}
$$

$$
\begin{aligned}
& -\frac{1}{2 \beta}\left\{(\tan (\beta t)-\beta t)-\frac{2 \sqrt{2} C_{2}}{\beta \cos (\beta t)}+\frac{C_{2}^{2} \tan (\beta t)}{\beta}\right\} \\
& +h t+\frac{\beta y^{2-2 \gamma}}{2 \rho^{2}(1-\gamma)}+\frac{y^{1-\gamma}}{\rho^{2}(1-\gamma)} \\
& -\frac{\beta \tan (\beta t) y^{2-2 \gamma}}{2 \rho^{2}(1-\gamma)^{2}} \pm \frac{y^{1-\gamma}}{\sqrt{2} \rho(1-\gamma) \cos (\beta t)} \\
& \left.\left\{-\sqrt{2} \sin (\beta t)+C_{2}\right\}\right] u(y, t) .
\end{aligned}
$$

We now construct the fundamental solutions of the bondpricing PDE (1). We have seen that there exist transformations (26) that reduce the PDE (1) to the classical heat equation. This result is used in the construction of the fundamental solution of bond-pricing equation.

The fundamental solution of the Cauchy problem for the heat equation is well-known and is given in barred coordinates by, see e.g [7],

$$
\bar{u}=\frac{1}{2 \sqrt{\bar{t} \pi}} \exp \left[-\frac{\bar{y}^{2}}{4 \bar{t}}\right] .
$$

We seek the fundamental solution for the PDE (1). The solution (27) is transformed by means of (26). We rewrite $\bar{u}$ from Eq. (26) as

$$
\bar{u}(y, t)=\left(\frac{1}{2} \rho^{2} y^{2 \gamma}\right)^{-\frac{1}{4}} \exp \left[P_{1}+Q_{1}\right] u(y, t) .
$$

By denoting

$$
\begin{gathered}
P_{1}=\exp \left[-\frac{1}{2} \ln \cos (\beta t)\right. \\
\left.-\frac{1}{2 \beta}\left\{(\tan (\beta t)-\beta t)-\frac{2 \sqrt{2} C_{2}}{\beta \cos (\beta t)}+\frac{C_{2}^{2} \tan (\beta t)}{\beta}\right\}+h t\right], \\
Q_{1}=\exp \left[\frac{\beta y^{2-2 \gamma}}{2 \rho^{2}(1-\gamma)}+\frac{y^{1-\gamma}}{\rho^{2}(1-\gamma)}-\frac{\beta \tan (\beta t) y^{2-2 \gamma}}{2 \rho^{2}(1-\gamma)^{2}}\right. \\
\left. \pm \frac{y^{1-\gamma}}{\sqrt{2} \rho(1-\gamma) \cos (\beta t)}\left\{-\sqrt{2} \sin (\beta t)+C_{2}\right\}\right]
\end{gathered}
$$

and making use of Eqs. (27) and (28), the solution $u(y, t)$ is written as

$$
u(y, t)=\frac{\left(\frac{1}{2} \rho^{2} y^{2 \gamma}\right)^{\frac{1}{4}}}{2 \sqrt{\bar{t} \pi}} \exp \left[-\frac{\bar{y}^{2}}{4 \bar{t}}-\left(P_{1}+Q_{1}\right)\right] .
$$

Finally, by substituting the values of $\bar{t}$ and $\bar{y}$ in Eq. (31), the solution becomes

$$
\begin{aligned}
u(y, t)= & \frac{\left(\frac{1}{2} \rho^{2} y^{2 \gamma}\right)^{\frac{1}{4}} \sqrt{\beta}}{2 \sqrt{C_{1} \pi \tan (\beta t)}} \exp \left[-\left(P_{1}+Q_{1}\right)\right] \times \\
& \exp \left[\frac { - \beta } { 4 C _ { 1 } \operatorname { t a n } ( \beta t ) } \left( \pm \frac{\sqrt{2 C_{1}} y^{1-\gamma}}{\cos (\beta t) \rho(1-\gamma)}-\frac{\sqrt{2 C_{1}}}{\beta \cos (\beta t)}\right.\right.
\end{aligned}
$$




$$
\left.\left.+\frac{\sqrt{C_{1}} C_{2} \tan (\beta t)}{\beta}+C_{3}\right)^{2}\right],
$$

where $P_{1}$ and $Q_{1}$ are defined in equations (29) and (30).

We now look for another form of solution for PDE (1). Another solution of the heat equation is given in the form of an infinite series [31]

$$
\bar{u}=\sum_{n=1}^{\infty} b_{n} \exp \left[-\left(\frac{n \pi}{L}\right)^{2} K \bar{t}\right] \sin \left(\frac{n \pi \bar{y}}{L}\right),
$$

which converges uniformly for $0 \leq y \leq L, t \geq 0$. Making use of the transformations (26), the solution $u(y, t)$ is written as

$$
\begin{aligned}
u(y, t)= & \left(\frac{1}{2} \rho^{2} y^{2 \gamma}\right)^{\frac{1}{4}} \exp \left[-\left(P_{1}+Q_{1}\right)\right] \sum_{n=1}^{\infty} b_{n} \\
& \exp \left[-\frac{n^{2} \pi^{2} K C_{1} \tan (\beta t)}{L^{2} \beta}\right] \\
& \sin \left(\frac { n \pi } { L } \left( \pm \frac{\sqrt{2 C_{1}} y^{1-\gamma}}{\cos (\beta t) \rho(1-\gamma)}-\frac{\sqrt{2 C_{1}}}{\beta \cos (\beta t)}\right.\right. \\
& \left.\left.+\frac{\sqrt{C_{1}} C_{2} \tan (\beta t)}{\beta}+C_{3}\right)\right),
\end{aligned}
$$

with $P_{1}$ and $Q_{1}$ defined as in equations (29) and (30).

\subsubsection{Case II: $\gamma=0, \alpha, \beta, \lambda$ and $\rho$ are arbitrary}

Making use of Theorem 5 and the coefficients defined in Eq. (20), we have

$$
\begin{aligned}
f(t)= & -\frac{\beta^{2}}{4}, \\
h(t)= & -\frac{\beta}{2}-\frac{\lambda^{2}}{2}-\frac{\alpha^{2}}{2 \rho^{2}}+\frac{\alpha \lambda}{\rho}, \\
\phi(t)= & \frac{C_{1}}{\beta} \tan (\beta t), \\
\beta(t)= & \frac{K \sqrt{C_{1}}}{\beta^{2}} \frac{1}{\cos (\beta t)}+\frac{\sqrt{C_{1}} C_{2}}{\beta} \tan (\beta t)+C_{3}, \\
g(t)= & -\frac{\rho}{\sqrt{2}}-\frac{\alpha \beta}{\rho \sqrt{2}}+\frac{\lambda \beta}{\sqrt{2}}, \\
v(t)= & \exp \left[-\frac{1}{2} \ln \cos (\beta t)-\frac{1}{4}\left\{\frac{K^{2}}{\beta^{3}}(\tan (\beta t)-\beta t)\right.\right. \\
& \left.\left.+\frac{2 C_{2} K}{\beta^{2}} \frac{1}{\cos (\beta t)}+\frac{C_{2}^{2}}{\beta} \tan (\beta t)\right\}+h t\right],
\end{aligned}
$$

where $C_{1}, C_{2}$ and $C_{3}$ are constants of integration and $K=-2 g(t)$. Using the values of the functions defined in Eq. (35) into Eq. (17) and simplifying, the transformations that reduce the bond-pricing equation (1) into the heat equation for Case II are:

$\bar{t}(y, t)=\frac{C_{1} \tan (\beta t)}{\beta}$,

$$
\begin{aligned}
\bar{y}(y, t)= & \pm \frac{\sqrt{2 C_{1}} y}{\rho \cos (\beta t)}-\frac{\sqrt{C_{1}} K}{\beta^{2} \cos (\beta t)}+\frac{\sqrt{C_{1}} C_{2} \tan (\beta t)}{\beta}+C_{3} \\
\bar{u}(y, t)= & \left(\frac{1}{2} \rho^{2}\right)^{-\frac{1}{4}} \exp \left[-\frac{1}{2} \ln \cos (\beta t)\right. \\
& -\frac{1}{4}\left\{\frac{K^{2}}{\beta^{3}}(\tan (\beta t)-\beta t)+\frac{2 C_{2} K}{\beta^{2} \cos (\beta t)}+\frac{C_{2}^{2} \tan (\beta t)}{\beta}\right\} \\
& +h t+\frac{(\alpha-\lambda \rho) y}{\rho^{2}}+\frac{\beta y^{2}}{2 \rho^{2}}-\frac{\beta \tan (\beta t) y^{2}}{2 \rho^{2}} \\
& \left. \pm \frac{y}{\sqrt{2} \rho \cos (\beta t)}\left\{\frac{K}{\beta} \sin (\beta t)+C_{2}\right\}\right] u(y, t) .
\end{aligned}
$$

We now construct the fundamental solutions for this case. We have seen that there exist transformations (36) that reduce the PDE (1) to the classical heat equation for Case II. This result is used in the construction of the fundamental solution of our bond-pricing equation.

The fundamental solution of the Cauchy problem for the heat equation is well-known and is given in barred coordinates by

$$
\bar{u}=\frac{1}{2 \sqrt{\bar{t} \pi}} \exp \left[-\frac{\bar{y}^{2}}{4 \bar{t}}\right] .
$$

Now the solution (37) is transformed by means of transformations (36). We rewrite $\bar{u}$ from Eq. (36) as

$$
\bar{u}(y, t)=\left(\frac{1}{2} \rho^{2}\right)^{-\frac{1}{4}} \exp \left[P_{2}+Q_{2}\right] u(y, t) .
$$

By denoting

$$
\begin{gathered}
P_{2}=\exp \left[h t-\frac{1}{2} \ln \cos (\beta t)\right. \\
\left.-\frac{1}{4}\left\{\frac{K^{2}}{\beta^{3}}(\tan (\beta t)-\beta t)+\frac{2 C_{2} K}{\beta^{2} \cos (\beta t)}+\frac{C_{2}^{2} \tan (\beta t)}{\beta}\right\}\right], \\
Q_{2}=\exp \left[\frac{(\alpha-\lambda \rho) y}{\rho^{2}}+\frac{\beta y^{2}}{2 \rho^{2}}-\frac{\beta \tan (\beta t) y^{2}}{2 \rho^{2}}\right. \\
\left. \pm \frac{y}{\sqrt{2} \rho \cos (\beta t)}\left\{\frac{K}{\beta} \sin (\beta t)+C_{2}\right\}\right]
\end{gathered}
$$

With the use of Eqs. (37) and (38), the solution $u(y, t)$ is written as

$$
u(y, t)=\frac{\left(\frac{1}{2} \rho^{2}\right)^{\frac{1}{4}}}{2 \sqrt{\bar{t} \pi}} \exp \left[-\frac{\bar{y}^{2}}{4 \bar{t}}\right] \exp \left[-\left(P_{2}+Q_{2}\right)\right] .
$$

Finally, by substituting the values of $\bar{t}$ and $\bar{y}$ from Eq. (36) into Eq. (41), the solution becomes

$$
\begin{aligned}
u(y, t)= & \frac{\left(\frac{1}{2} \rho^{2}\right)^{\frac{1}{4}} \sqrt{\beta}}{2 \sqrt{C_{1} \pi \tan (\beta t)}} \exp \left[-\left(P_{2}+Q_{2}\right)\right] \times \\
& \exp \left[-\frac{\beta}{4 C_{1} \tan (\beta t)}\left( \pm \frac{\sqrt{2 C_{1}} y}{\rho \cos (\beta t)}-\frac{\sqrt{C_{1}} K}{\beta^{2} \cos (\beta t)}\right.\right.
\end{aligned}
$$




$$
\left.\left.+\frac{\sqrt{C_{1}} C_{2} \tan (\beta t)}{\beta}+C_{3}\right)^{2}\right],
$$

where $P_{2}$ and $Q_{2}$ are defined in equations (39) and (40).

We now seek another form of the solution for the PDE

(1) for Case II. Another solution of the heat equation is given in the form of an infinite series

$$
\bar{u}=\sum_{n=1}^{\infty} b_{n} \exp \left[-\left(\frac{n \pi}{L}\right)^{2} \kappa \bar{t}\right] \sin \left(\frac{n \pi \bar{y}}{L}\right),
$$

which converges uniformly for $0 \leq y \leq L, t \geq 0$. Making use of the transformations (36), the solution for $u(y, t)$ is written as:

$$
\begin{aligned}
u(y, t)= & \left(\frac{1}{2} \rho^{2}\right)^{\frac{1}{4}} \exp \left[-\left(P_{2}+Q_{2}\right)\right] \\
& \sum_{n=1}^{\infty} b_{n} \exp \left[-\frac{n^{2} \pi^{2} \kappa C_{1} \tan (\beta t)}{L^{2} \beta}\right] \times \\
& \sin \left(\frac { n \pi } { L } \left( \pm \frac{\sqrt{2 C_{1}} y}{\rho \cos (\beta t)}-\frac{\sqrt{C_{1}} K}{\beta^{2} \cos (\beta t)}\right.\right. \\
& \left.\left.+\frac{\sqrt{C_{1}} C_{2} \tan (\beta t)}{\beta}+C_{3}\right)\right),
\end{aligned}
$$

where $P_{2}$ and $Q_{2}$ are given as in equations (39) and (40).

\subsubsection{Case III: $\gamma=\frac{1}{2}, \alpha=\frac{\rho^{2}}{4}, \lambda, \beta$ and $\rho$ are arbitrary}

For this case, the values of the functions defined in Theorem 5 are:

$$
\begin{aligned}
f(t) & =-\frac{\rho^{2}}{8}-\frac{\beta^{2}}{16}, \\
h(t) & =-\frac{\lambda^{2}}{2}-\frac{\beta}{4}, \\
\phi(t) & =\frac{C_{1}}{p^{\star}} \tan \left(p^{\star} t\right), \\
\beta(t) & =\frac{B \sqrt{C_{1}}}{\left(p^{\star}\right)^{2}} \frac{1}{\cos \left(p^{\star} t\right)}+\frac{\sqrt{C_{1}} C_{2}}{p^{\star}} \tan \left(p^{\star} t\right)+C_{3}, \\
g(t) & =\frac{\lambda \beta}{2 \sqrt{2}}, \\
v(t) & =\exp \left[-\frac{1}{2} \ln \cos \left(p^{\star} t\right)-\frac{1}{4}\left\{\frac{B^{2}}{\left(p^{\star}\right)^{3}}\left(\tan \left(p^{\star} t\right)-p^{\star} t\right)\right.\right. \\
& \left.\left.+\frac{2 C_{2} B}{\left(p^{\star}\right)^{2}} \frac{1}{\cos \left(p^{\star} t\right)}+\frac{C_{2}^{2}}{p^{\star}} \tan \left(p^{\star} t\right)\right\}+h t\right],
\end{aligned}
$$

where $C_{1}, C_{2}$ and $C_{3}$ are constants of integration, $B=$ $-\lambda \beta / \sqrt{2}$ and $p^{*}=\left(\sqrt{\rho^{2}+\beta^{2} / 2}\right) / \sqrt{2}$. The transformations that reduce the bond-pricing equation (1) into the heat equation for Case III are given by

$$
\bar{t}(y, t)=\frac{C_{1}}{p^{\star}} \tan \left(p^{\star} t\right),
$$

$$
\begin{aligned}
\bar{y}(y, t) & = \pm \frac{\sqrt{2 C_{1}} y}{\rho \cos \left(p^{\star} t\right)}+\frac{\sqrt{C_{1}} B}{\left(p^{\star}\right)^{2} \cos \left(p^{\star} t\right)} \\
& +\frac{\sqrt{C_{1}} C_{2} \tan \left(p^{\star} t\right)}{p^{\star}}+C_{3}, \\
\bar{u}(y, t) & =\left(\frac{1}{2} \rho^{2} y\right)^{-\frac{1}{4}} \exp \left[-\frac{1}{2} \ln \cos \left(p^{\star} t\right)\right. \\
& -\frac{1}{4}\left\{\frac{B^{2}}{\left(p^{\star}\right)^{3}}\left(\tan \left(p^{\star} t\right)-p^{\star} t\right)+\frac{2 C_{2} B}{\left(p^{\star}\right)^{2} \cos \left(p^{\star} t\right)}\right. \\
& \left.+\frac{C_{2}^{2} \tan \left(p^{\star} t\right)}{p^{\star}}\right\}+h t+\frac{\ln y}{4}+\frac{\beta y}{\rho^{2}}-\frac{2 \lambda \sqrt{y}}{\rho} \\
& -\frac{p^{\star} \tan \left(p^{\star} t\right) y}{\rho^{2}} \\
& \left. \pm \frac{2 \sqrt{2} \sqrt{y}}{\rho \cos \left(p^{\star} t\right)}\left\{\frac{B}{p^{\star}} \sin \left(p^{\star} t\right)+C_{2}\right\}\right] u(y, t) .
\end{aligned}
$$

Following the same methodology adopted for the previous two cases, the fundamental solutions of the bond-pricing PDE corresponding to the transformation (46) are deduced as

$$
\begin{aligned}
u(y, t)= & \frac{\left(\frac{1}{2} \rho^{2} y\right)^{\frac{1}{4}} \sqrt{p^{\star}}}{2 \sqrt{C_{1} \pi \tan \left(p^{\star} t\right)}} \exp \left[-\left(P_{3}+Q_{3}\right)\right] \times \\
& \exp \left[C_{3}-\frac{p^{\star}}{4 C_{1} \tan \left(p^{\star} t\right)}\left( \pm \frac{\sqrt{2 C_{1}} y}{\rho \cos \left(p^{\star} t\right)}\right.\right. \\
& \left.\left.+\frac{\sqrt{C_{1}} B}{\left(p^{\star}\right)^{2} \cos \left(p^{\star} t\right)}+\frac{\sqrt{C_{1}} C_{2} \tan \left(p^{\star} t\right)}{p^{\star}}\right)^{2}\right], \\
u(y, t)= & \left.\frac{1}{2} \rho^{2} y\right)^{\frac{1}{4}} \exp \left[-\left(P_{3}+Q_{3}\right)\right] \\
& \sum_{n=1}^{\infty} b_{n} \exp \left[-\frac{n^{2} \pi^{2} \kappa C_{1} \tan \left(p^{\star} t\right)}{L^{2} p^{\star}}\right] \\
& \sin \left(\frac { n \pi } { L } \left( \pm \frac{\sqrt{2 C_{1}} y}{\rho \cos \left(p^{\star} t\right)}\right.\right. \\
& \left.\left.+\frac{\sqrt{C_{1}} B}{\left(p^{\star}\right)^{2} \cos \left(p^{\star} t\right)}+\frac{\sqrt{C_{1}} C_{2} \tan \left(p^{\star} t\right)}{p^{\star}}+C_{3}\right)\right),
\end{aligned}
$$

where $P_{3}$ and $Q_{3}$ are defined as

$$
\begin{aligned}
P_{3}= & \exp \left[-\frac{1}{2} \ln \cos \left(p^{\star} t\right)-\frac{1}{4}\left\{\frac{B^{2}}{\left(p^{\star}\right)^{3}}\left(\tan \left(p^{\star} t\right)-p^{\star} t\right)\right.\right. \\
& \left.\left.+\frac{2 C_{2} B}{\left(p^{\star}\right)^{2} \cos \left(p^{\star} t\right)}+\frac{C_{2}^{2} \tan \left(p^{\star} t\right)}{p^{\star}}\right\}+h t\right],
\end{aligned}
$$




$$
\begin{aligned}
Q_{3}= & \exp \left[\frac{\ln y}{4}+\frac{\beta y}{\rho^{2}}-\frac{2 \lambda \sqrt{y}}{\rho}-\frac{p^{\star} \tan \left(p^{\star} t\right) y}{\rho^{2}}\right. \\
& \left. \pm \frac{2 \sqrt{2} \sqrt{y}}{\rho \cos \left(p^{\star} t\right)}\left\{\frac{B}{\left(p^{\star}\right)} \sin \left(p^{\star} t\right)+C_{2}\right\}\right] .
\end{aligned}
$$

\subsubsection{Case IV: $\gamma=\frac{1}{2}, \alpha=\frac{3 \rho^{2}}{4}, \lambda=0, \beta$ and $\rho$ are arbitrary}

Finally for this case, we deduce the following values of the functions defined in Theorem 5.

$$
\begin{aligned}
& f(t)=-\frac{\rho^{2}}{8}-\frac{\beta^{2}}{16}, \\
& h(t)=-\frac{3 \beta}{4}, \\
& \phi(t)=\frac{C_{1}}{p^{\star}} \tan \left(p^{\star} t\right), \\
& \beta(t)=C_{1} \int \frac{d t}{\cos \left(p^{\star} t\right) \ln \cos \left(p^{\star} t\right)}, \\
& g(t)=0, \\
& v(t)=\exp \left[\frac{1}{2} \ln \cos \left(p^{\star} t\right)-\frac{1}{4} \int \frac{d t}{\left[\ln \cos \left(p^{\star} t\right)\right]^{2}}+h t\right],
\end{aligned}
$$

with $C_{1}, C_{2}$ and $C_{3}$ are the constants of integration and $p^{*}=\sqrt{\rho^{2}+\beta^{2} / 2} / \sqrt{2}$. The corresponding transformations are found as

$$
\begin{aligned}
\bar{t}(y, t) & =\frac{C_{1}}{p^{\star}} \tan \left(p^{\star} t\right), \\
\bar{y}(y, t) & = \pm \frac{2 \sqrt{2 C_{1} \sqrt{y}}}{\rho \cos \left(p^{\star} t\right)}+C_{1} \int \frac{d t}{\cos \left(p^{\star} t\right) \ln \cos \left(p^{\star} t\right)}, \\
\bar{u}(y, t) & =\left(\frac{1}{2} \rho^{2} y\right)^{-\frac{1}{4}} \exp \left[\frac{1}{2} \ln \cos \left(p^{\star} t\right)\right. \\
& -\frac{1}{4} \int \frac{d t}{\left[\ln \cos \left(p^{\star} t\right)\right]^{2}}+h t+\frac{3 \ln y}{4}+\frac{\beta}{\rho^{2}} \\
& \left.-\frac{p^{\star} \tan \left(p^{\star} t\right) y}{\rho^{2}} \pm \frac{C_{3} 2 \sqrt{2} \sqrt{y}}{\rho \ln \cos \left(p^{\star} t\right)}\right] u(y, t) .
\end{aligned}
$$

The fundamental solutions of the bond-pricing equation for Case IV are given by

$$
\begin{aligned}
u(y, t) & =\frac{\left(\frac{1}{2} \rho^{2} y\right)^{\frac{1}{4}} \sqrt{p^{\star}}}{2 \sqrt{C_{1} \pi \tan \left(p^{\star} t\right)}} \exp \left[-\left(P_{4}+Q_{4}\right)\right] \times \\
& \exp \left[-\frac{p^{\star}}{4 C_{1} \tan \left(p^{\star} t\right)}\left( \pm \frac{2 \sqrt{2 C_{1}} \sqrt{y}}{\rho \cos \left(p^{\star} t\right)}\right.\right. \\
& \left.\left.+C_{1} \int \frac{d t}{\cos \left(p^{\star} t\right) \ln \cos \left(p^{\star} t\right)}\right)^{2}\right],
\end{aligned}
$$

$$
\begin{aligned}
u(y, t) & =\left(\frac{1}{2} \rho^{2} y\right)^{\frac{1}{4}} \exp \left[-\left(P_{4}+Q_{4}\right)\right] \\
& \sum_{n=1}^{\infty} b_{n} \exp \left[-\frac{n^{2} \pi^{2} \kappa C_{1} \tan \left(p^{\star} t\right)}{L^{2} p^{\star}}\right] \times \\
& \sin \left(\frac { n \pi } { L } \left( \pm \frac{2 \sqrt{2 C_{1}} \sqrt{y}}{\rho \cos \left(p^{\star} t\right)}\right.\right. \\
& \left.\left.+C_{1} \int \frac{d t}{\cos \left(p^{\star} t\right) \ln \cos \left(p^{\star} t\right)}\right)\right),
\end{aligned}
$$

where $P_{4}$ and $Q_{4}$ are:

$$
\begin{gathered}
P_{4}=\exp \left[\frac{1}{2} \ln \cos \left(p^{\star} t\right)-\frac{1}{4} \int \frac{d t}{\left[\ln \cos \left(p^{\star} t\right)\right]^{2}}+h t\right], \\
Q_{4}=\exp \left[\frac{3 \ln y}{4}+\frac{\beta}{\rho^{2}}-\frac{p^{\star} \tan \left(p^{\star} t\right) y}{\rho^{2}} \pm \frac{2 C_{3} \sqrt{2} \sqrt{y}}{\rho \ln \cos \left(p^{\star} t\right)}\right] .
\end{gathered}
$$

\subsection{Reduction to second Lie canonical form}

We now focus on the instance where the bond pricing equation (1) is equivalent to the second Lie canonical form (11). Since the PDE (1) is autonomous, the invariant condition (12) reduces to

$$
\begin{aligned}
& 20 L_{X}+10\left[|a|^{\frac{1}{2}} L_{X}\right]_{X} \int \frac{d x}{|a|^{\frac{1}{2}}}+\left[|a|^{\frac{1}{2}}\left[|a|^{\frac{1}{2}} L_{X}\right]_{X}\right]_{X}\left(\int \frac{d x}{|a|^{\frac{1}{2}}}\right)^{2} \\
& =0
\end{aligned}
$$

Two cases arise in the evaluation of the invariant equation (57). This is due to the evaluation of the integral in (57). We therefore end up with two possible values for parameter $\gamma$, viz. $\gamma=1$ and $\gamma \neq 1$.

For $\gamma=1$, the condition (57) becomes

$$
\begin{aligned}
& 10 \rho^{2}+10 \alpha \rho^{2} y^{-2}-40 \alpha^{2} y^{-3}-10 \alpha \beta y^{-2}+10 \alpha \lambda \rho y^{-2} \\
& +5 \rho^{2} \ln y-5 \alpha \rho^{2} y^{-2} \ln y+40 \alpha^{2} y^{-3} \ln y+5 \alpha \beta y^{-2} \ln y \\
& -5 \alpha \lambda \rho y^{-2} \ln y+\frac{1}{2} \rho^{2} \ln ^{2} y+\frac{1}{2} \alpha \rho^{2} y^{-2} \ln ^{2} y \\
& -8 \alpha^{2} y^{-3} \ln ^{2} y-\frac{1}{2} \alpha \beta y^{-2} \ln ^{2} y+\frac{1}{2} \alpha \lambda \rho y^{-2} \ln ^{2} y=0 .
\end{aligned}
$$

The condition (58) implies $\rho=0$, which is physically not allowed. Thus we conclude that for $\gamma=1$, the bond-pricing PDE (1) is not reducible to the second Lie canonical form by point transformation. Now for $\gamma \neq 1$, the condition (57) is

$$
10 \rho^{2} \gamma(2 \gamma-1) y^{2 \gamma-2}+30 \lambda \rho^{3} \gamma(\gamma-1)^{3} y^{3 \gamma-4}
$$




$$
\begin{aligned}
& -30 \rho^{4} \gamma^{2}(\gamma-1)^{3} y^{4 \gamma-5}+60 \rho^{4} \gamma(\gamma-1)^{3} y^{4 \gamma-5} \\
& +10 \alpha \rho^{2} \gamma(\gamma-2)(2 \gamma-3) y^{2 \gamma-4}-20 \alpha^{2} \gamma(\gamma+1) y^{-3} \\
& +10 \alpha \beta \gamma(1-2 \gamma) y^{-2}-10 \alpha \lambda \rho \gamma(\gamma-2) y^{\gamma-3} \\
& +5 \rho^{2} \gamma \frac{(2 \gamma-1)}{(1-\gamma)}(3 \gamma-2) y^{2 \gamma-2}+60 \lambda \rho^{3} \gamma(1-\gamma)^{3} y^{3 \gamma-4} \\
& -75 \rho^{4} \gamma^{2}(1-\gamma)^{3} y^{4 \gamma-5}+150 \rho^{4} \gamma(1-\gamma)^{3} y^{4 \gamma-5} \\
& +5 \alpha \rho^{2} \gamma \frac{(\gamma-2)}{(1-\gamma)}(2 \gamma-3)(3 \gamma-4) y^{2 \gamma-4} \\
& -10 \alpha^{2} \gamma \frac{(\gamma+1)}{(1-\gamma)}(\gamma-3) y^{-3}+5 \alpha \beta \gamma \frac{(1-2 \gamma)}{(1-\gamma)}(\gamma-2) y^{-2} \\
& -5 \alpha \lambda \rho \gamma \frac{(\gamma-2)}{(1-\gamma)}(2 \gamma-3) y^{\gamma-3} \\
& +\frac{1}{2} \rho^{2} \gamma \frac{(2 \gamma-1)}{(1-\gamma)^{2}}(3 \gamma-2)(4 \gamma-3) y^{2 \gamma-2} \\
& +30 \lambda \rho^{3} \gamma(\gamma-1)^{3} y^{3 \gamma-4}-45 \rho^{4} \gamma^{2}(\gamma-1)^{3} y^{4 \gamma-5} \\
& +90 \rho^{4} \gamma(\gamma-1)^{3} y^{4 \gamma-5}+\frac{1}{2} \alpha \rho^{2} \gamma \frac{(\gamma-2)}{(1-\gamma)^{2}}(2 \gamma-3) \\
& (3 \gamma-4)(4 \gamma-5) y^{2 \gamma-4}-2 \alpha^{2} \gamma \frac{(\gamma+1)}{(1-\gamma)^{2}}(\gamma-3)(\gamma-2) y^{-3} \\
& +\frac{1}{2} \alpha \beta \gamma \frac{(1-2 \gamma)}{(1-\gamma)^{2}}(\gamma-2)(2 \gamma-3) y^{-2} \\
& -\frac{1}{2} \alpha \lambda \rho \gamma \frac{(\gamma-2)}{(1-\gamma)^{2}}(2 \gamma-3)(3 \gamma-4) y^{\gamma-3}=0 .
\end{aligned}
$$

The analysis of the invariant condition (59) is similar to that for the heat invariant criterion (24). We deduce the following three cases:

Case A: $\gamma=2, \alpha=0, \lambda, \beta$ and $\rho$ are arbitrary

Case B: $\gamma=3 / 2, \alpha=0, \beta, \lambda$ and $\rho$ are arbitrary

Case C: $\gamma=1 / 2, \alpha, \lambda, \beta$ and $\rho$ are arbitrary

We find that Case $\mathrm{A}$ is missing in the work [28]. For the Cases $B$ and $C$ partial results are obtained in [28]. In fact for Cases B and C four nontrivial symmetries were only found for $\lambda=0$ in [28]. Therefore, for all the cases we found above, our results are new.

\subsection{Transformation formulae and fundamental solutions}

Here we find the transformations which reduce the bondpricing PDE (1) to second Lie canonical form for each of the three cases deduced in the previous section.

\subsubsection{Case A: $\gamma=2, \alpha=0, \lambda, \beta$ and $\rho$ are arbitrary}

Making use of the coefficients defined in Eq. (20) in Eqs. (18) and (19) in Theorem 5, we have

$$
f(t)=0,
$$

$$
\begin{aligned}
h(t) & =-\frac{\lambda^{2}}{2}, \\
\phi(t) & =-\frac{C_{1}}{t}+C_{2}, \\
\beta(t) & =0, \\
g(t) & =0, \\
v(t) & =\sqrt{t} \exp \left[C_{4} t+C_{3}\right],
\end{aligned}
$$

where $C_{1}, C_{2}, C_{3}$ and $C_{4}$ are constants of integration. By using values of the functions from Eq. (60) into Eq. (17) and simplifying, the transformations that reduce the parabolic equation (1) into the second Lie canonical form are:

$$
\begin{aligned}
\bar{t}(y, t) & =-\frac{C_{1}}{t}+C_{2}, \\
\bar{y}(y, t) & = \pm \frac{\sqrt{2 C_{1}}}{\rho t} \frac{1}{y}, \\
\bar{u}(y, t) & =\left(\frac{1}{2} \rho^{2} y^{4}\right)^{-\frac{1}{4}} \exp \\
& {\left[C_{4} t+C_{3}-\frac{\beta}{2 \rho^{2} y^{2}}+\frac{\lambda}{\rho y}-\frac{1}{2 \rho t y^{2}}\right] u(y, t) . }
\end{aligned}
$$

We now construct the fundamental solutions of the bond-pricing PDE (1). We have seen that there exist transformations (61) that reduce the PDE (1) to the second Lie canonical form, given by

$$
\bar{u}_{\bar{t}}=\bar{u}_{\bar{y} \bar{y}}+\frac{A}{\bar{y}^{2}} \bar{u},
$$

We first derive the solution for the PDE (62) and then use the transformations (61) for the construction of the fundamental solution of the bond-pricing equation.

In order to derive the solution for PDE (62), we make use of the method of separation of variables. We assume the solution of PDE (62) is of the form

$$
\bar{u}(\bar{y}, \bar{t})=F(\bar{y}) G(\bar{t}) .
$$

Making use of Eq. (63) into Eq. (62) and separating, we obtain

$$
\begin{aligned}
\frac{d G}{d \bar{t}}+\lambda G & =0, \\
\frac{d^{2} F}{d \bar{y}^{2}}+\left(\frac{A}{\bar{y}^{2}}+\lambda\right) F & =0,
\end{aligned}
$$

where $\lambda \geq 0$ is the separation constant.

Case A1: when $\lambda=0$

For this case the general solution of ODEs (64) and (65) are:

$$
\begin{aligned}
G(\bar{t}) & =B_{1}, \\
F(\bar{y}) & =B_{2} \bar{y}^{\frac{1}{2}(1-\sqrt{1-4 A})}+B_{3} \bar{y}^{\frac{1}{2}(1+\sqrt{1-4 A})},
\end{aligned}
$$


with $B_{1}, B_{2}$ and $B_{3}$ are constants of integration. Thus the solution of PDE (62) is written as

$$
\bar{u}(\bar{y}, \bar{t})=B_{1}\left[B_{2} \bar{y}^{\frac{1}{2}(1-\sqrt{1-4 A})}+B_{3} \bar{y}^{\frac{1}{2}(1+\sqrt{1-4 A})}\right] .
$$

In order to find the fundamental solution for the PDE (1), the solution (68) is transformed by means of (61). Making use of Eqs. (68) and (61), the solution $u(y, t)$ is written as

$$
\begin{aligned}
& u(y, t)=\left(\frac{1}{2} \rho^{2} y^{4}\right)^{\frac{1}{4}} \exp \left[-\left(R_{1}+S_{1}\right)\right] \\
& B_{1}\left[B_{2} \bar{y}^{\frac{1}{2}(1-\sqrt{1-4 A})}+B_{3} \bar{y}^{\frac{1}{2}(1+\sqrt{1-4 A})}\right],
\end{aligned}
$$

where

$$
\begin{aligned}
& R_{1}=C_{4} t+C_{3}, \\
& S_{1}=-\frac{\beta}{2 \rho^{2} y^{2}}+\frac{\lambda}{\rho y}-\frac{1}{2 \rho t y^{2}} .
\end{aligned}
$$

Finally, with the substitution the values of $\bar{y}$ from (61) into Eq. (69), the solution becomes

$$
\begin{aligned}
u(y, t) & =\left(\frac{1}{2} \rho^{2} y^{4}\right)^{\frac{1}{4}} \exp \left[-\left(R_{1}+S_{1}\right)\right] \times \\
B_{1} & {\left[B_{2}\left( \pm \frac{\sqrt{2 C_{1}}}{\rho t} \frac{1}{y}\right)^{\frac{1}{2}(1-\sqrt{1-4 A})}\right.} \\
& \left.+B_{3}\left( \pm \frac{\sqrt{2 C_{1}}}{\rho t} \frac{1}{y}\right)^{\frac{1}{2}(1+\sqrt{1-4 A})}\right] .
\end{aligned}
$$

Case A2: when $\lambda>0$

For this case the general solution of ODE (64) is

$$
G(\bar{t})=B_{1} \exp (-\lambda \bar{t}) .
$$

The general solution of the homogenous equation (65) is (see, e.g., [32])

$$
\begin{aligned}
& F(\bar{y})=\left[B_{2} \sqrt{\bar{y}} \mathcal{\partial}\left(\frac{1}{2} \sqrt{1-4 A} ; \sqrt{\lambda} \bar{y}\right)\right. \\
& \left.+B_{3} \sqrt{\bar{y}} y\left(\frac{1}{2} \sqrt{1-4 A} ; \sqrt{\lambda} \bar{y}\right)\right],
\end{aligned}
$$

where $B_{1}, B_{2}$ and $B_{3}$ are constants of integration and $\mathcal{J}$ and $y$ are Bessel functions of the first and second kind, respectively (see, e.g., [33]). Thus the separable solution of the second Lie canonical form (62) is given by

$$
\begin{aligned}
& \bar{u}(\bar{y}, \bar{t})=B_{1} \exp (-\lambda \bar{t})\left[B_{2} \sqrt{\bar{y}} \mathcal{J}\left(\frac{1}{2} \sqrt{1-4 A} ; \sqrt{\lambda} \bar{y}\right)\right. \\
& \left.+B_{3} \sqrt{\bar{y}} y\left(\frac{1}{2} \sqrt{1-4 A} ; \sqrt{\lambda} \bar{y}\right)\right] .
\end{aligned}
$$

To find the fundamental solution for the PDE (1), the solution (75) is transformed by means of (61). Making use of Eqs. (75) and (61), the solution $u(y, t)$ is written as

$$
u(y, t)=B_{1}\left(\frac{1}{2} \rho^{2} y^{4}\right)^{\frac{1}{4}} \exp \left[-\left(R_{1}+S_{1}\right)\right] \times
$$

$$
\begin{aligned}
& \exp (-\lambda \bar{t})\left[B_{2} \sqrt{\bar{y}} \mathcal{J}\left(\frac{1}{2} \sqrt{1-4 A} ; \sqrt{\lambda} \bar{y}\right)\right. \\
& \left.+B_{3} \sqrt{\bar{y}} y\left(\frac{1}{2} \sqrt{1-4 A} ; \sqrt{\lambda} \bar{y}\right)\right],
\end{aligned}
$$

where $R_{1}$ and $S_{1}$ are given in Eqs. (70) and (71). Finally, making use of $\bar{t}$ and $\bar{y}$ from (61) in Eq. (76), the solution $u(y, t)$ takes the form

$$
\begin{aligned}
u(y, t)= & B_{1}\left(\frac{1}{2} \rho^{2} y^{4}\right)^{\frac{1}{4}} \exp \left[-\left(R_{1}+S_{1}\right)-\lambda\left(-\frac{C_{1}}{t}+C_{2}\right)\right] \times \\
& {\left[B_{2} \sqrt{ \pm \frac{\sqrt{2 C_{1}}}{\rho t} \frac{1}{y}} \mathcal{\partial}\left(\frac{1}{2} \sqrt{1-4 A} ; \sqrt{\lambda} \frac{ \pm \sqrt{2 C_{1}}}{\rho t} \frac{1}{y}\right)\right.} \\
& \left.+B_{3} \sqrt{ \pm \frac{\sqrt{2 C_{1}}}{\rho t}} \frac{1}{y} y\left(\frac{1}{2} \sqrt{1-4 A} ; \sqrt{\lambda} \frac{ \pm \sqrt{2 C_{1}}}{\rho t} \frac{1}{y}\right)\right] .
\end{aligned}
$$

\subsubsection{Case B: $\gamma=\frac{3}{2}, \alpha=0, \beta, \lambda$ and $\rho$ are arbitrary}

For this case, the values of the functions defined in Theorem 5 are:

$$
\begin{aligned}
f(t) & =-\frac{\beta^{2}}{16}, \\
h(t) & =\beta, \\
\phi(t) & =\frac{2 C_{1} \tan \left(\frac{\beta}{2} t\right)}{\beta}, \\
\beta(t) & =0, \\
g(t) & =0, \\
v(t) & =\sqrt{\cos \left(\frac{\beta}{2} t\right)} \exp \left[\beta t+C_{2}\right] .
\end{aligned}
$$

The corresponding transformations are given by

$$
\begin{aligned}
& \bar{t}(y, t)=\frac{2 C_{1} \tan \left(\frac{\beta}{2} t\right)}{\beta}, \\
& \bar{y}(y, t)= \pm \frac{2 \sqrt{2 C_{1}}}{\rho \cos \left(\frac{\beta}{2} t\right)} \frac{1}{\sqrt{y}}, \\
& \bar{u}(y, t)=\left(\frac{1}{2} \rho^{2} y^{3}\right)^{-\frac{1}{4}} \sqrt{\cos \left(\frac{\beta}{2} t\right)} \\
& \exp \left[\beta t+C_{2}-\frac{\beta}{\rho^{2} y}-\frac{\beta \tan \left(\frac{\beta}{2} t\right)}{\rho^{2} y}\right] u(y, t) .
\end{aligned}
$$

We now construct the fundamental solutions for this case. We have seen that there exist transformations (79) that reduce the PDE (1) to the second canonical form for Case B. This result is used in the construction of the fundamental solution of the bond-pricing equation. Again we have two cases to discuss. 
Case B1: when $\lambda=0$ (separation constant)

For this case the general solution of the second Lie canonical form (62) is given by

$$
\bar{u}(\bar{y}, \bar{t})=B_{1}\left[B_{2} \bar{y}^{\frac{1}{2}(1-\sqrt{1-4 A})}+B_{3} \bar{y}^{\frac{1}{2}(1+\sqrt{1-4 A})}\right] .
$$

In order to find the fundamental solution for the PDE (1), we have to transform the solution (80) by means of (79). Using equations Eqs. (80) and (79), the solution $u(y, t)$ is written as

$$
\begin{aligned}
u(y, t) & =B_{1}\left(\frac{1}{2} \rho^{2} y^{3}\right)^{\frac{1}{4}}\left(\cos \left(\frac{\beta}{2} t\right)\right)^{-\frac{1}{2}} \exp \left[-\left(R_{2}+S_{2}\right)\right] \times \\
& {\left[B_{2} \bar{y}^{\frac{1}{2}(1-\sqrt{1-4 A})}+B_{3} \bar{y}^{\frac{1}{2}(1+\sqrt{1-4 A})}\right], }
\end{aligned}
$$

with

$$
\begin{aligned}
& R_{2}=\beta t+C_{2}, \\
& S_{2}=-\frac{\beta}{\rho^{2} y}\left\{1+\tan \left(\frac{\beta}{2} t\right)\right\} .
\end{aligned}
$$

Finally, using the values of $\bar{y}$ from (79), the solution (81) takes the form

$$
\begin{aligned}
u(y, t)= & B_{1}\left(\frac{1}{2} \rho^{2} y^{3}\right)^{\frac{1}{4}}\left(\cos \left(\frac{\beta}{2} t\right)\right)^{-\frac{1}{2}} \exp \left[-\left(R_{2}+S_{2}\right)\right] \times \\
& {\left[B_{2}\left( \pm \frac{2 \sqrt{2 C_{1}}}{\rho \cos \left(\frac{\beta}{2} t\right)} \frac{1}{\sqrt{y}}\right)^{\frac{1}{2}(1-\sqrt{1-4 A})}\right.} \\
& \left.+B_{3}\left( \pm \frac{2 \sqrt{2 C_{1}}}{\rho \cos \left(\frac{\beta}{2} t\right)} \frac{1}{\sqrt{y}}\right)^{\frac{1}{2}(1+\sqrt{1-4 A})}\right] .
\end{aligned}
$$

Case B2: when $\lambda>0$ (separation constant)

For this case the separable solution of the second Lie canonical form (62) is given by

$$
\begin{aligned}
& \bar{u}(\bar{y}, \bar{t})=B_{1} \exp (-\lambda \bar{t})\left[B_{2} \sqrt{\bar{y}} \mathcal{J}\left(\frac{1}{2} \sqrt{1-4 A} ; \sqrt{\lambda} \bar{y}\right)\right. \\
& \left.+B_{3} \sqrt{\bar{y}} y\left(\frac{1}{2} \sqrt{1-4 A} ; \sqrt{\lambda} \bar{y}\right)\right]
\end{aligned}
$$

where $B_{1}, B_{2}$ and $B_{3}$ are constants of integration and $\mathcal{J}$ and $y$ are Bessel functions of the first and second kind, respectively. Under the transformations (79), the solution $u(y, t)$ is given by

$$
\begin{aligned}
u(y, t)= & B_{1}\left(\frac{1}{2} \rho^{2} y^{3}\right)^{\frac{1}{4}}\left(\cos \left(\frac{\beta}{2} t\right)\right)^{-\frac{1}{2}} \exp \left[-\left(R_{2}+S_{2}\right)\right] \times \\
& \exp (-\lambda \bar{t})\left[B_{2} \sqrt{\bar{y}} \jmath\left(\frac{1}{2} \sqrt{1-4 A} ; \sqrt{\lambda} \bar{y}\right)\right.
\end{aligned}
$$

$$
\left.+B_{3} \sqrt{\bar{y}} y\left(\frac{1}{2} \sqrt{1-4 A} ; \sqrt{\lambda} \bar{y}\right)\right],
$$

where $R_{2}$ and $S_{2}$ are given in Eqs. (82) and (83). Finally, by making use of the values of $\bar{t}$ and $\bar{y}$ from (79) into Eq. (86), the solution $u(y, t)$ takes the form

$$
\begin{aligned}
u(y, t) & =B_{1}\left(\frac{1}{2} \rho^{2} y^{3}\right)^{\frac{1}{4}}\left(\cos \left(\frac{\beta}{2} t\right)\right)^{-\frac{1}{2}} \\
& \exp \left[-\left(R_{2}+S_{2}\right)-\lambda\left(\frac{2 C_{1} \tan \left(\frac{\beta}{2} t\right)}{\beta}\right)\right] \times \\
& {\left[B_{2} \sqrt{ \pm \frac{2 \sqrt{2 C_{1}}}{\rho \cos \left(\frac{\beta}{2} t\right)} \frac{1}{\sqrt{y}}}\right.} \\
& \mathcal{\partial}\left(\frac{1}{2} \sqrt{1-4 A} ; \sqrt{\lambda} \frac{ \pm 2 \sqrt{2 C_{1}}}{\rho \cos \left(\frac{\beta}{2} t\right)} \frac{1}{\sqrt{y}}\right) \\
& +B_{3} \sqrt{\frac{2 \sqrt{2 C_{1}}}{\rho \cos \left(\frac{\beta}{2} t\right)} \frac{1}{\sqrt{y}}} \\
& \left.y\left(\frac{1}{2} \sqrt{1-4 A} ; \sqrt{\lambda} \frac{ \pm 2 \sqrt{2 C_{1}}}{\rho \cos \left(\frac{\beta}{2} t\right)} \frac{1}{\sqrt{y}}\right)\right] .
\end{aligned}
$$

where $R_{2}$ and $S_{2}$ are defined in Eqs. (82) and (83)

\subsubsection{Case $\mathrm{C}: \gamma=\frac{1}{2}, \alpha, \lambda, \beta$ and $\rho$ are arbitrary}

Finally for this Case $\mathrm{C}$, the values of the functions defined in Theorem 5 are given by

$$
\begin{aligned}
f(t) & =-\frac{\beta^{2}}{16}-\frac{\rho^{2}}{8}, \\
h(t) & =-\frac{\alpha \beta}{\rho^{2}}, \\
\phi(t) & =\frac{C_{1}}{p^{\star}} \tan \left(p^{\star} t\right), \\
\beta(t) & =0, \\
g(t) & =0, \\
v(t) & =\sqrt{\cos \left(p^{\star} t\right)} \exp \left[-\frac{\alpha \beta}{\rho^{2}} t+C_{2}\right],
\end{aligned}
$$

with $C_{1}$ and $C_{2}$ being constants of integration and $p^{*}=$ $\sqrt{\rho^{2}+\beta^{2} / 2} / \sqrt{2}$. The transformations for reduction of PDE (1) into the second Lie canonical form for Case $\mathrm{C}$ are:

$$
\begin{aligned}
& \bar{t}(y, t)=\frac{C_{1}}{p^{\star}} \tan \left(p^{\star} t\right), \\
& \bar{y}(y, t)= \pm \frac{2 \sqrt{2 C_{1}}}{\rho \cos \left(p^{\star} t\right)} \sqrt{y},
\end{aligned}
$$




$$
\begin{aligned}
\bar{u}(y, t)= & \left(\frac{1}{2} \rho^{2} y\right)^{-\frac{1}{4}} \sqrt{\cos p^{\star} t} \\
& \exp \left[C_{2}-\frac{\alpha \beta}{\rho^{2}} t+\frac{\alpha \ln y}{\rho^{2}}+\frac{\beta y}{\rho^{2}}-\frac{p^{\star} y}{\rho^{2}} \tan \left(p^{\star} t\right)\right] \\
& u(y, t) .
\end{aligned}
$$

By using the same methodology adopted in the previous two cases, the fundamental solution of the bond-pricing equation (1) under the transformations (89) for the case of separation constant $\lambda=0$ is given by

$$
\begin{aligned}
u(y, t) & =B_{1}\left(\frac{1}{2} \rho^{2} y\right)^{\frac{1}{4}}\left(\cos p^{\star} t\right)^{-\frac{1}{2}} \exp \left[-\left(R_{3}+S_{3}\right)\right] \times \\
& {\left[B_{2}\left( \pm \frac{2 \sqrt{2 C_{1}}}{\rho \cos \left(p^{\star} t\right)} \sqrt{y}\right)^{\frac{1}{2}(1-\sqrt{1-4 A})}\right.} \\
& \left.+B_{3}\left( \pm \frac{2 \sqrt{2 C_{1}}}{\rho \cos \left(p^{\star} t\right)} \sqrt{y}\right)^{\frac{1}{2}(1+\sqrt{1-4 A})}\right] .
\end{aligned}
$$

For the case when the separation constant $\lambda>0$, the solution of PDE (1) becomes

$$
\begin{aligned}
u(y, t) & =B_{1}\left(\frac{1}{2} \rho^{2} y\right)^{\frac{1}{4}}\left(\cos p^{\star} t\right)^{-\frac{1}{2}} \\
& \exp \left[-\left(R_{3}+S_{3}\right)-\lambda\left(\frac{C_{1}}{p^{\star}} \tan \left(p^{\star} t\right)\right)\right] \times \\
& {\left[B_{2} \sqrt{ \pm \frac{2 \sqrt{2 C_{1}}}{\rho \cos \left(p^{\star} t\right)} \sqrt{y}}\right.} \\
& \mathcal{J}\left(\frac{1}{2} \sqrt{1-4 A} ; \sqrt{\lambda} \frac{ \pm 2 \sqrt{2 C_{1}}}{\rho \cos \left(p^{\star} t\right)} \sqrt{y}\right) \\
& +B_{3} \sqrt{ \pm \frac{2 \sqrt{2 C_{1}}}{\rho \cos \left(p^{\star} t\right)} \sqrt{y}} \\
& \left.y\left(\frac{1}{2} \sqrt{1-4 A} ; \sqrt{\lambda} \frac{ \pm 2 \sqrt{2 C_{1}}}{\rho \cos \left(p^{\star} t\right)} \sqrt{y}\right)\right],
\end{aligned}
$$

where $\mathcal{J}$ and $y$ are the Bessel functions of first and second kind and $R_{3}$ and $S_{3}$ are given by

$$
\begin{aligned}
& R_{3}=-\frac{\alpha \beta}{\rho^{2}} t+C_{2}, \\
& S_{3}=\frac{\alpha \ln y}{\rho^{2}}+\frac{\beta y}{\rho^{2}}-\frac{p^{*} y}{\rho^{2}} \tan \left(p^{\star} t\right) .
\end{aligned}
$$

Remark. Note that from the condition (14) given in Theorem 3, we have $\partial J / \partial t=0$, excluding when the invariant conditions (8) and (12) apply (as for these conditions we already have the values of parameter $\lambda$ ). In this case the bond-pricing PDE (1) can be reduced to the third Lie canonical form (13) for which it admits two nontrivial symmetries. Finally, we do not have the condition (16) being satisfied by the bond-pricing PDE (1) as equation (1) is autonomous.

\section{Solution via the heat equation Cauchy problem}

In this section, we construct the fundamental solution of the Cauchy problem for the bond-pricing model:

$$
\begin{gathered}
\frac{\partial u}{\partial t}+\frac{1}{2} \rho^{2} y^{2 \gamma} \frac{\partial^{2} u}{\partial y^{2}}+\left(\alpha+\beta y-\lambda \rho y^{\gamma}\right) \frac{\partial u}{\partial y}-y u=0, \\
u(y, 0)=1 .
\end{gathered}
$$

In the previous sections, we found the equivalence transformations which map the bond-pricing model to the classical heat equation for different cases of the parameters involved. Here we utilize these transformations for the construction of the fundamental solutions of the underlying model. We first consider:

Case I: $\gamma=2, \alpha=0, \lambda=-1 / \rho, \beta$ and $\rho$ are arbitrary

The transformations are derived in Eq. (26) which reduce the Cauchy problem (94) and (95) for the bondpricing model into the standard heat equation Cauchy problem

$$
\begin{aligned}
\frac{\partial \bar{u}}{\partial \bar{t}} & =\frac{\partial^{2} \bar{u}}{\partial \bar{y}^{2}}, \\
\bar{u}(\bar{y}, 0) & =\phi(\bar{y}),
\end{aligned}
$$

for some function $\phi$. Provided $\phi$ is "well-behaved". The solution to (96) and (97) is well-known [34, 35] and is given by

$$
\bar{u}(\bar{y}, \bar{t})=\frac{1}{2 \sqrt{t} \pi} \int_{-\infty}^{+\infty} \phi(\zeta) \exp \left[-\frac{(\bar{y}-\zeta)^{2}}{4 \bar{t}}\right] d \zeta .
$$

To transform the solution (98) into the solution of (94) and (95), we start by writing $u(y, t)$ from Eq. (26) in the form

$$
u(y, t)=\left(\frac{1}{2} \rho^{2} y^{2 \gamma}\right)^{\frac{1}{4}} \exp \left[-\left(P_{1}+Q_{1}\right)\right] \bar{u}(\bar{y}, \bar{t}) .
$$

where $P_{1}$ and $Q_{1}$ are defined in Eqs. (29) and (30). Substituting $\bar{u}(\bar{y}, \bar{t})$ from Eq. (98) into Eq. (99), we obtain

$$
\begin{aligned}
u(y, t) & =\left(\frac{1}{2} \rho^{2} y^{2 \gamma}\right)^{\frac{1}{4}} \exp \left[-\left(P_{1}+Q_{1}\right)\right] \frac{1}{2 \sqrt{\bar{t} \pi}} \int_{-\infty}^{+\infty} \phi(\zeta) \\
& \exp \left[-\frac{(\bar{y}-\zeta)^{2}}{4 \bar{t}}\right] d \zeta,
\end{aligned}
$$


where $\phi(\zeta)$ is found from Eq. (26). By keeping in mind the condition (95), we have

$$
\begin{aligned}
& \phi(\zeta)=\left(\frac{1}{2} \rho^{2} \zeta^{2 \gamma}\right)^{-\frac{1}{4}} \\
& \exp \left[-\frac{2 \sqrt{2} C_{2}}{\beta}+\frac{\beta \zeta^{2-2 \gamma}}{2 \rho^{2}(1-\gamma)}+\frac{\zeta^{1-\gamma}}{\rho^{2}(1-\gamma)} \pm \frac{\zeta^{1-\gamma} C_{2}}{\sqrt{2} \rho(1-\gamma)}\right] .
\end{aligned}
$$

Finally, substituting the values of $\bar{y}$ and $\bar{t}$ from Eq. (26) into Eq. (100), the solution becomes

$$
\begin{aligned}
u(y, t)= & \left(\frac{1}{2} \rho^{2} y^{2 \gamma}\right)^{\frac{1}{4}} \exp \left[-\left(P_{1}+Q_{1}\right)\right] \frac{\sqrt{\beta}}{2 \sqrt{C_{1} \tan (\beta t) \pi}} \times \\
& \int_{-\infty}^{+\infty} \phi(\zeta) \exp \left[\frac { - \beta } { 4 C _ { 1 } \operatorname { t a n } ( \beta t ) } \left(C_{3} \pm \frac{\sqrt{2 C_{1}} y^{1-\gamma}}{\cos (\beta t) \rho(1-\gamma)}\right.\right. \\
& \left.\left.-\frac{\sqrt{2 C_{1}}}{\beta \cos (\beta t)}+\frac{\sqrt{C_{1}} C_{2} \tan (\beta t)}{\beta}-\zeta\right)^{2}\right] d \zeta,
\end{aligned}
$$

where $\phi(\zeta)$ is defined in (101). One can deduce the value of the integral in (102) in terms of the Kummer confluent hypergeometric functions.

For Case II, the equivalence transformations for the reduction into heat equation Cauchy problem have already been derived in Eq. (36). Under the equivalence transformations the corresponding closed-form solutions for the Cauchy problem (94) and (95) of the bond-pricing model is given by

$$
\begin{aligned}
u(y, t)= & \left(\frac{1}{2} \rho^{2}\right)^{\frac{1}{4}} \exp \left[-\left(P_{2}+Q_{2}\right)\right] \frac{\sqrt{\beta}}{2 \sqrt{C_{1} \pi \tan (\beta t)}} \times \\
& \int_{-\infty}^{+\infty} \phi(\zeta) \exp \left[\frac { - \beta } { 4 C _ { 1 } \operatorname { t a n } ( \beta t ) } \left(C_{3} \pm \frac{\sqrt{2 C_{1}} y}{\rho \cos (\beta t)}\right.\right. \\
& \left.\left.-\frac{\sqrt{C_{1}} K}{\beta^{2} \cos (\beta t)}+\frac{\sqrt{C_{1}} C_{2} \tan (\beta t)}{\beta}-\zeta\right)^{2}\right] d \zeta,
\end{aligned}
$$

with

$\phi(\zeta)=\left(\frac{1}{2} \rho^{2}\right)^{-\frac{1}{4}} \exp \left[\frac{2 C_{2} K}{\beta^{2}}+\frac{(\alpha-\lambda \rho) \zeta}{\rho^{2}}+\frac{\beta \zeta^{2}}{2 \rho^{2}} \pm \frac{\zeta C_{2} K}{\beta \sqrt{2} \rho}\right]$, where $P_{2}$ and $Q_{2}$ are defined in Eqs. (39) and (40). For Case III and Case IV one can derive the closed-form Cauchy solutions in a similar manner.

\section{Concluding remarks}

In this paper we used the invariant approach on the bondpricing partial differential equation (1). The approach provides a wide class of fundamental solutions of the PDE in question. The classification of the bond-pricing equation relied on Lie's classification results of parabolic equations and the use of transformation formulae. We have shown that the Lie symmetry algebra of this equation depends upon the values of the parameters $\lambda, \alpha, \gamma, \beta$ and $\rho$. We found various cases for which the bond-pricing equation can be mapped to the first and second Lie canonical forms. We have shown the Cases I to IV for reduction of the bondpricing equation into the classical heat equation depending upon the various values of parameters involved. For each of these cases, we computed the required equivalence transformations to the heat equation. The transformations derived made it possible to obtain fundamental solutions of the bond-pricing equation from that of the heat equation. We have also found new cases of the parameters for which the bond-pricing equation has four nontrivial symmetries and hence can be mapped to the second Lie canonical form. For all such values of these arbitrary constants for reduction into the second Lie canonical form, we have obtained transformation relations and fundamental solutions of the bond-pricing equation. Finally, using this approach, the closed-form solution of the Cauchy problem for the bond-pricing model with suitable choice of boundary conditions is derived for the first time.

It is noteworthy that the authors introduced the invariance principle for boundary value problems to find fundamental solutions of the Cauchy problems for linear parabolic PDEs. The above-mentioned method is a combination of application of Lie symmetries and the use of the theory of generalized functions. That is, if the boundary value problem is invariant under a group, then the fundamental solution is found among the functions invariant under that group. Also the invariant approach discussed here can be applied to other parabolic models which transform not only to the heat equation but to other canonical forms as well. We also mention the future research investigations of the possible usefulness of this method in the wonderful world of financial mathematics.

Acknowledgement: The authors would like to thank Dr T. Motsepa for fruitful discussions. A. Fatima thanks the the North-West University, Mafikeng Campus, South Africa for postdoctoral fellowship award for 2018.

\section{References}

[1] Gazizov R.K., Ibragimov N.H., Lie symmetry analysis of differential equations in finance, Nonlinear Dynam., 1998, 17(4), 387407. 
[2] Tamizhmani K.M., Krishnakumar K., Leach P.G.L., Algebraic resolution of equations of the Black-Scholes type with arbitrary time-dependent parameters, Appl. Math. Comput., 2014, 247, 115-124.

[3] Motsepa T., Aziz T., Fatima A., Khalique C.M., Algebraic aspects of evolution partial differential equation arising in the study of constant elasticity of variance model from financial mathematics, Open Phys., 2018, 16, 31-36.

[4] Motsepa T., Khalique C.M., Molati M., Group classification of a general bond-option pricing equation of mathematical finance, Abstract Appl. Anal., 2014, Volume 2014, Article ID 709871, 10 pages.

[5] Lo C.F., Hui C.H., Valuation of financial derivatives with timedependent parameters, Quant. Fin., 2001, 1, 73-78.

[6] Sinkala W., On the generation of arbitrage-free stock price models using Lie symmetry analysis, Comput. Math. Appl., 2016, 72, 1386-1393.

[7] Pooe C.A., Mahomed F.M., Wafo Soh C., Fundamental solutions for zero-coupon bond pricing models, Nonlinear Dynam., 2004, 36, 69-76.

[8] Basov S., Lie groups of PDEs and their application to the multidimensional screening problems, in: Econometric Society 2004 Australasian Meetings, vol. 44, (2004).

[9] Liu Y., Wang D.S., Symmetry analysis of the option pricing model with dividend yield from financial market, App. Math. Lett., 2011, 24, 481-486.

[10] Wang Z., Wang L., Wang D.S., Jin Y., Optimal system, symmetry reductions and new closed form solutions for the geometric average Asian options, Appl. Math. Comput., 2014, 226, 598-605.

[11] Davison A.H., Mamba S., Symmetry methods for option pricing, Commun. Nonlinear Sci. Numer. Simulat., 2017, 47, 421-425.

[12] Dimas S., Andriopoulos K., Tsoubelis D., Leach P.G.L., Complete specification of some PDEs that arise in financial mathematics, J. Nonlinear Math. Phys., 2009, 16, 73-92.

[13] Naicker V., Andriopoulos K., Leach P.G.L., Symmetry reductions of a Hamilton-Jacobi-Bellman equation arising in financial mathematics, J. Nonlinear Math. Phys., 2005, 12(2), 268 283.

[14] Polidoro S., A nonlinear PDE in Mathematical Finance, in: F. Brezzi, A. Buffa, S. Corsaro, A. Murli (Eds.), Numerical Mathematics and Advanced Application, Springer, 2003.

[15] Sinkala W., Leach P.G.L., O'Hara J.G., An optimal system and group-invariant solutions of the Cox-Ingersoll-Ross pricing equation, Appl. Math. Comput., 2008, 201, 95-107.

[16] Zhou S., Xiao L., An Application of symmetry approach to finance: Gauge symmetry in finance, Symmetry., 2010, 2(4), 1763-1775.

[17] Bordag L.A., Yamshchikov I.P., Optimization problem for a portfolio with an illiquid asset: Lie group analysis, J. Math. Anal. Appl., 2017, 453, 668-699.

[18] Bachelier L., Theorie de la speculation, Annales Scientifiques de l'Ecole Normale Superieure., 1900, 3, 21-86.

[19] Merton R.C., Optimum consumption and portfolio rules in a continuous time model, J. Economic Thr., 1971, 3(4), 373-413.

[20] Black F., Scholes M., The pricing of options and corporate liabilities, J. Political Eco., 1973, 81, 637-654.

[21] Vasicek 0., An equilibrium characterization of the term structure, J. Financial Eco., 1977, 5, 177-188.

[22] Black F., Karasinski P., Bond and option pricing when short rates are lognormal, Financial Analysts J., 1991, 47, 52-59.
[23] Heath D., Jarrow R., Morton A., Bond pricing and the term structure of interest rates: a new methodology for contingent claims valuation, Econometrica, 1992, 60, 77-105.

[24] Cox J.C., Ingersoll J.E., Ross S.A., An intertemporal general equilibrium model of asset prices, Econometrica, 1985, 53, 363-384.

[25] Brennan M.J., Schwartz E.S., Analyzing convertible bonds, J. Financial Quantitative Anal., 1980, 15, 907-929.

[26] Dothan L., On the term structure of interest rates, J. Financial Econ., 1978, 6, 59-69.

[27] Johnpillai I.K., Mahomed F.M., Singular invariant equation for the $(1+1)$ Fokker- Planck equation, J. Physics A: Mathematical and General, 2001, 34, 11033-11051.

[28] Mahomed F.M., Complete invariant characterization of scalar linear (1+1) parabolic equations, J. Nonlinear Math. Phys., 2008, $15,112-123$.

[29] Sinkala W., Leach P.G.L., O’Hara J.G., Invariance properties of a general bond-pricing equation, J. Differential Equ., 2008, 244, 2820-2835.

[30] Olver P.J., Applications of Lie Groups to Differential Equations, Graduate Texts in Mathematics, Vol. 107, Springer-Verlag, New York, 1993.

[31] Lie S., On integration of a class of linear partial differential equations by means of definite integrals, Archiv for Mathematik og Naturvidenskab, [in German], Reprinted in S. Lie,Gesammelte Abhadlundgen, 3 paper XXXV, 1881, 3, 328368.

[32] Myint-U T.Y.N., Partial Differential Equations of Mathematical Physics, Amercian Elsevier Publishing Company, INC. New York, 1973.

[33] Polyanin A.D., Zaitsev V.F., Handbook of Exact Solutions for Ordinary Differential Equations, CRC Press, Boca Raton, FL, 1995.

[34] Abramowitz M., Stegun I.A., Handbook of Mathematical Functions, New York, 1965.

[35] Kallianpur G., Karandikar R.L., Introduction to option pricing theory. Boston: Birkhäuser, 2000.

[36] Hadamard J., Lectures on Cauchy's Problem in Linear Partial Differential Equations, Yale University Press, New Haven, Connecticut, 1923. 